\title{
KULEUVEN
}

\begin{tabular}{||l|l|}
\hline Citation & $\begin{array}{l}\text { Dixian Zhao, Shailesh Kulkarni, Patrick Reynaert, (2012), } \\
\text { A 60GHz Outphasing Transmitter in 40nm CMOS with 15.6dBm Output } \\
\text { Power } \\
\text { IEEE ISSCC Dig. Tech. Papers, Feb. 2012, pp. 170-171. }\end{array}$ \\
\hline Archived version & $\begin{array}{l}\text { Author manuscript: the content is identical to the content of the published } \\
\text { paper, but without the final typesetting by the publisher }\end{array}$ \\
\hline Published version & $\begin{array}{l}\text { http://ieeexplore.ieee.org/xpl/articleDetails.jsp?tp=\&arnumber=6176963\&quer } \\
\text { yText\%3Ddixian+zhao }\end{array}$ \\
\hline Conference homepage & $\begin{array}{l}\text { http://isscc.org/index.html } \\
\text { Author contact }\end{array}$ \\
\hline $\begin{array}{l}\text { email dixian.zhao@esat.kuleuven.be } \\
\text { phone number }+32(0) 16321149\end{array}$ \\
\hline
\end{tabular}

(article begins on next page) 


\section{A 60GHz Outphasing Transmitter in 40nm CMOS with $15.6 \mathrm{dBm}$ Output Power}

\author{
Dixian Zhao, Shailesh Kulkarni, Patrick Reynaert
}

\section{K.U. Leuven, Leuven, Belgium}

One of the important technical problems related to $60 \mathrm{GHz}$ CMOS transmitters is the poor average efficiency when transmitting amplitude- and phase-modulated signals. The cause of this low efficiency is the required back-off from the output $1 \mathrm{~dB}$ compression point $\left(\mathrm{P}_{1 \mathrm{~dB}}\right)$ to meet EVM and transmit spectrum mask specifications. A conventional power amplifier (PA) only provides maximum efficiency near the saturated output power $\left(P_{S A T}\right)$. For a $6 \mathrm{~dB}$ back-off from $P_{1 \mathrm{~dB}}$, the output power and PAE of the state-of-the-art PAs remain below $9 \mathrm{dBm}$ and $5 \%$, respectively [1,2].

This paper presents a $60 \mathrm{GHz}$ transmitter (TX) based on the outphasing technique [3]. It avoids amplifying variable-envelope signals and reconstructs the modulated signals by vector summing two constant-amplitude phase-modulated signals using an on-chip power combiner. The proposed design proves to have higher linear output power with better average efficiency compared to existing $60 \mathrm{GHz}$ solutions.

As shown in the system diagram of Fig. 9.5.1, the outphasing TX requires constant-amplitude phase-modulated baseband signals $S_{11}(t), S_{12}(t)$ and $S_{01}(t)$ $S_{O_{2}}(t)$ where $\theta(t)$ indicates the modulated phase and the outphasing angie $\varphi(t)$ determines the instantaneous amplitude $\left(\varphi(t)=\cos ^{-1}[a(t) / 2 A]\right)$. The baseband signals are upconverted by the $1 / Q$ mixer to generate the desired outphasing signals at PA inputs (i.e., $\left.S_{1}(t)=A \sin \left[\omega_{c} t+\theta(t)+\varphi(t)\right], S_{2}(t)=A \sin \left[\omega_{c} t+\theta(t)-\varphi(t)\right]\right)$. As the two PAs only need to amplify constant-envelope signals, they keep operating in saturation and thus achieve high power efficiency. At the output, the power combiner sums the amplified signals and reconstructs the amplitude modulation. Since the outputs of the two PAs are coupled through a transformer-based combiner, their load impedance increases when the outphasing angle increases (i.e., when the output power decreases) [3]. This load modulation effect reduces the PA power consumption and is another major benefit of outphasing. Note that this TX can also operate in conventional direct-conversion mode when $S_{11}(t)=$ $S_{12}(t)=I(t)$ and $S_{O 1}(t)=S_{Q 2}(t)=Q(t)$. The measurement results of both operating modes (outphasing and conventional) will be compared.

The TX consists of a power combiner, two PAs, I/Q upconversion mixers, polyphase filters (PPF), LO buffers and single-ended-to-differential (SE/D) baseband amplifiers. Figure 9.5 .2 shows the implementation of the $1 / Q$ double-balanced mixer, which is tuned at $60 \mathrm{GHz}$ by a transformer and $35 \mu \mathrm{m}$ interconnects. As this TX uses a direct-conversion architecture, carrier feedthrough may arise due to asymmetries in the circuit, which can be alleviated by employing a commongate (CG) buffer amplifier. In contrast to a common-source (CS) stage, the CG stage loads the mixer with 10 times lower input impedance and hence reduces the LO-to-RF coupling (from LO port to the input terminal of the buffer) by 5 to $10 \mathrm{~dB}$ in the $60 \mathrm{GHz}$ band. The $\mathrm{I} / \mathrm{Q}$ LOS are generated by a two-stage PPF to achieve an image rejection ratio (IRR) of $25 \mathrm{~dB}$ over the entire $60 \mathrm{GHz}$ band. As shown in the floorplan of the PPF, all the Rs and Cs are laid out in a way to simplify the routing and improve the matching $\left(C_{1}=30 \mathrm{fF}, \mathrm{C}_{2}=34 \mathrm{fF}, \mathrm{R}=75 \Omega\right)$.

The PA incorporates two unit PAs with a power combiner at the output (see Fig. 9.5.1). In the design, the neutralization technique is adopted by cross-connecting the interdigitated metal-oxide-metal capacitors between the drain and gate terminals of the differential stage to improve the stability and reverse isolation. The transformer-based combiner has a loss of $1.2 \mathrm{~dB}$ and sums the outphasing vectors at the TX output. Since the outphasing angle between these two vectors varies over time, a combiner as in [2] cannot be used. Instead, a combiner with two separate transformers is developed for outphasing operation. As shown in Fig. 9.5.4, the primary side of the combiner has two pairs of differential ports excited by outphasing signals while the center taps provide DC access. The unwanted magnetic coupling of adjacent windings is minimized by orthogonal placement of the two coils. This combiner has a $-1 \mathrm{~dB} B W$ of $20 \mathrm{GHz}$ which guarantees correct outphasing operation.
The TX is fabricated in a 40nm digital CMOS technology. In the design, inductors and transformers are carefully filled with metal dummies, resulting in a performance degradation of only $2 \%$ according to field simulation. Mismatch between the two outphasing signals $S_{1}(t)$ and $S_{2}(t)$ (see Fig. 9.5.1) is a potential cause of amplitude and phase distortion. Therefore, special attention is paid to the floorplan of the TX and the physical layout, to minimize the asymmetries in baseband, in $1 / Q L O$ distribution and between the $S_{1}(t)$ and $S_{2}(t)$ signal paths. Figure 9.5.7 shows the die micrograph and the TX measures $0.96 \mathrm{~mm}^{2}$. The measurements are performed on a high-frequency probe station with the $D C$ and low-frequency pads wire-bonded to a PCB. In the measurements, no predistortion or equalization is applied.

Figure 9.5.3 shows that the TX has a measured conversion gain of $26 \mathrm{~dB}$ and the $-3 \mathrm{~dB}$ RF BW is more than $7 \mathrm{GHz}(59.5$ to $67 \mathrm{GHz})$. The IF BW is measured by varying the baseband input frequency, which exceeds $3.6 \mathrm{GHz}$ and is sufficient to accommodate outphasing signais for $60 \mathrm{GHz}$. In the 57 to $66 \mathrm{GHz}$ band the $\mathrm{P}_{\text {SAT }}$. measured with a power meter, varies between 13.8 and $15.6 \mathrm{dBm}$ while the PAE (only PA) is higher than $17 \%$ with a peak value of $25 \%$ at $60 \mathrm{GHz}$. The IRR is better than $20 \mathrm{~dB}$ in the $60 \mathrm{GHz}$ band. The transmitted QPSK spectrum with a symbol rate of $1.76 \mathrm{GS} / \mathrm{s}$ and a roll-off factor of 0.35 meets the spectral mask of the IEEE802.15.3c and WiGig standards at an output power of $10.5 \mathrm{dBm}$.

The two major benefits of the TX in outphasing mode are demonstrated in Fig. 9.5.4, which shows the measured output power and the PA power consumption for both outphasing and conventional direct-conversion modes. First, the TX in conventional mode starts to saturate at a $P_{1 \mathrm{~dB}}$ of $13 \mathrm{dBm}$ and shows a $P_{S A T}$ of $15.6 \mathrm{dBm}$ while the outphasing $\mathrm{TX}$ is able to operate linearly up to the maximum output power of $15.6 \mathrm{dBm}$ ( $\left.P_{\text {lin max }}=P_{S A T}\right)$. Consequently, the outphasing $T X$ does not need to operate at back-off when processing variable envelope signals. Second, the outphasing PA consumes less DC power because of the load modulation effect. Therefore, the outphasing mode is able to achieve higher average output power $\left(\mathrm{P}_{\mathrm{AVG}}\right)$ and better average efficiency with complex modulation.

The benefits of the outphasing mode for efficiency and linear output power are further confirmed by EVM measurements with varying envelope QPSK and 8PSK signals at $62 \mathrm{GHz}$. For a roll-off factor of 0.35 (raised-cosine filter), the peak-toaverage power ratio (PAPR) of both QPSK and 8PSK is $4.9 \mathrm{~dB}$. Figure 9.5 .5 shows that the outphasing mode achieves $>13 \mathrm{dBm} P_{A V G}$ and $>15 \%$ average $P A$ efficiency $\left(\eta_{\mathrm{AVG}}=\mathrm{P}_{\mathrm{AVG}} / \mathrm{P}_{\mathrm{DC}, \mathrm{PA}}\right)$ for both QPSK and 8PSK at the EVM of $-25 \mathrm{~dB}$. It performs two times better than the conventional direct-conversion mode in $P_{A V G}$ and $\eta_{\text {AVG }}$ for the same EVM.

Figure 9.5 .6 compares the measured performance with other $60 \mathrm{GHz}$ transmitters, clearly showing the improved efficiency and higher linear output power of the presented outphasing TX.

\section{Acknowledgments:}

This work is funded by the Catrene PANAMA project, the IWT and ST-Ericsson Zaventem, Belgium. The authors thank F. Daenen from K.U.Leuven and D. JooS, T. Finateu, W. Philibert from ST-Ericsson for their technical support.

\section{References:}

[1] C. Liang and B. Razavi, "Transmitter linearization by beamforming," IEEE J. Solid-State Circuits, vol. 46, no. 9, pp.1956-1969, Sept. 2011.

[2] J. Chen and A.M. Niknejad, "A compact 1V $18.6 \mathrm{dBm} 60 \mathrm{GHz}$ power amplifier in 65nm CMOS ," ISSCC Dig. Tech. Papers, pp. 432-433, Feb. 2011.

[3] H. Chireix, "High power outphasing modulation," Proc. IRE, vol.23, no. 11, pp. 1370-1392, Nov. 1935.

[4] K. Okada et al., "A 60GHz 16QAM/8PSK/QPSK/BPSK direct-conversion transceiver for IEEE 802.," ISSCC Dig. Tech. Papers, pp. 160-161, Feb. 2011. 


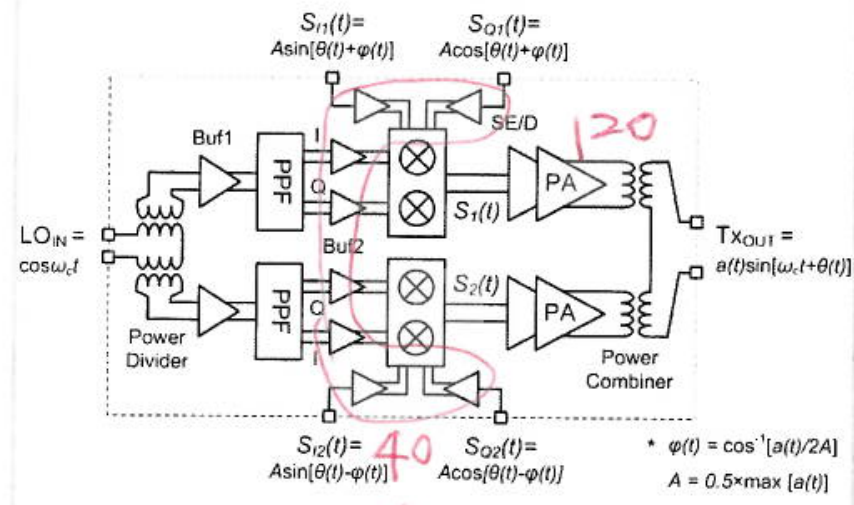

$$
\text { A } 40
$$

Figure 9.5.1: System diagram of the outphasing transmitter.
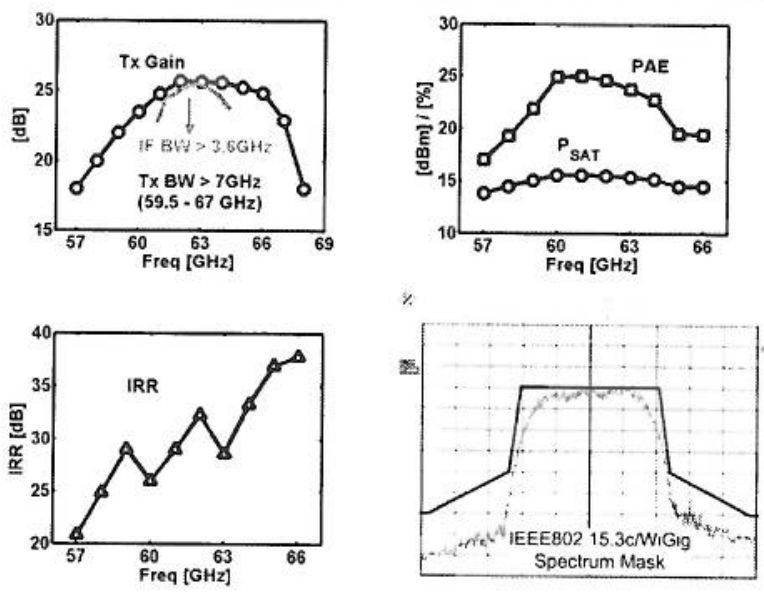

Figure 9.5.3: Measured TX conversion gain, IF BW, $P_{\text {SAT }}$, PAE (PA) and IRR in $60 \mathrm{GHz}$ band, and the TX QPSK spectrum at $\mathrm{f}_{\mathrm{C}}$ of $62.64 \mathrm{GHz}$.
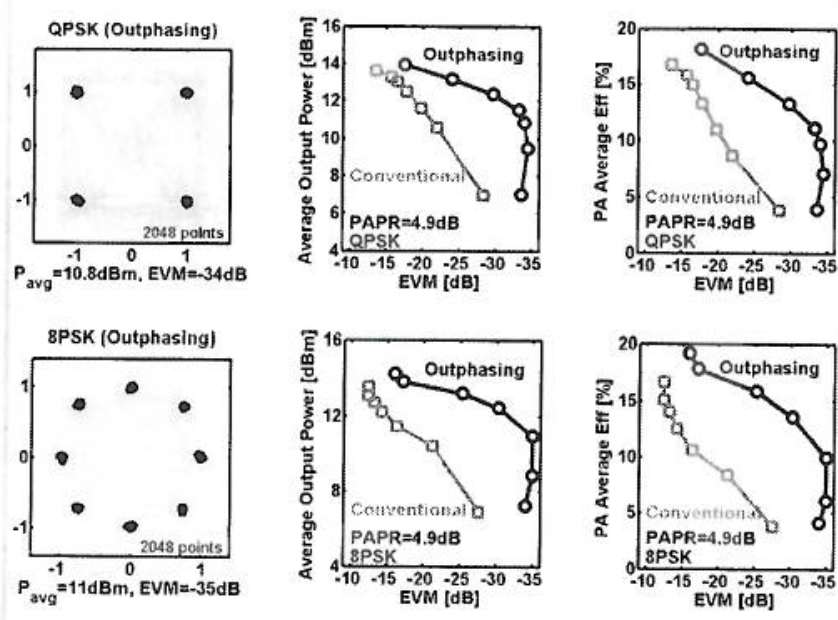

Figure 9.5.5: Measured constellations of QPSK and 8PSK in outphasing mode, and comparison with conventional mode.

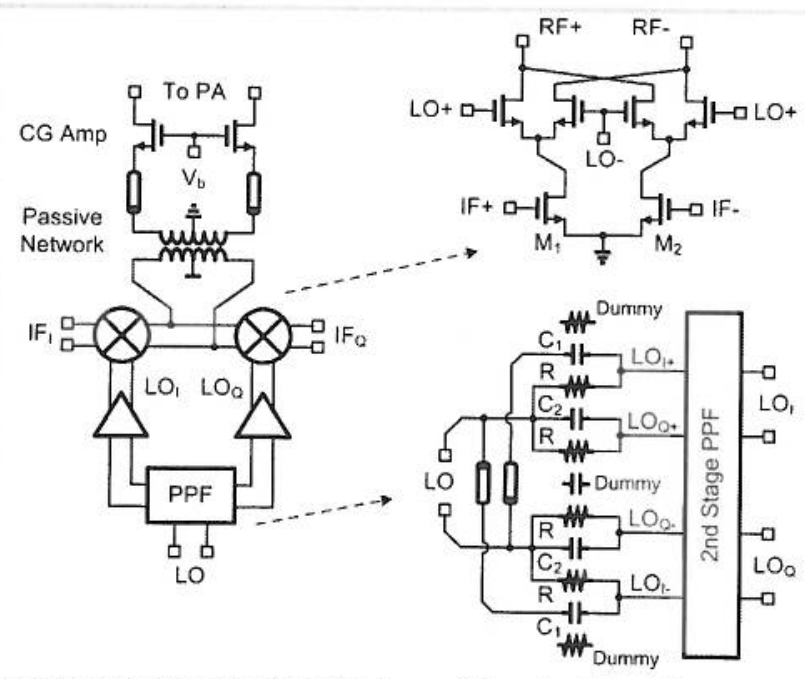

Figure 9.5.2: Schematic of the $\mathrm{I} / \mathrm{Q}$ mixer and floorplan of the PPF.

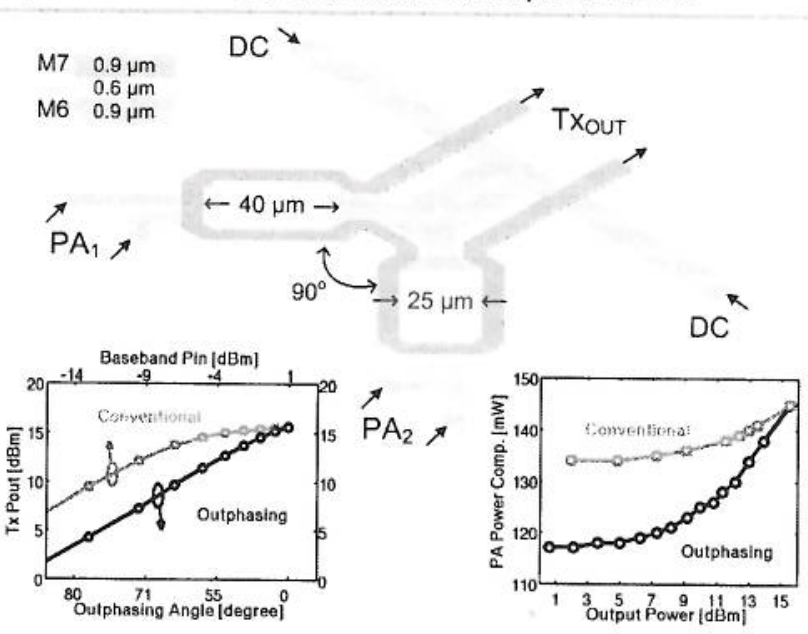

Figure 9.5.4: Layout of the power combiner, measured $P_{0 U T}$ and $P A$ power consumption in outphasing and conventional modes.

\begin{tabular}{|c|c|c|c|c|}
\hline & This Work & This Work & [1] & {$[4]$} \\
\hline Architecture & $\begin{array}{l}\text { Outphasing, } \\
\text { power comb. }\end{array}$ & $\begin{array}{l}\text { Direct-conv:, } \\
\text { power comb. }\end{array}$ & $\begin{array}{c}\text { Outphasing. } \\
\text { beamforning }\end{array}$ & Direct-cons: \\
\hline$P_{\text {len, } 1 \text { nax }}[\mathrm{dBm}]$ & 15.6 & $13\left(\mathrm{P}_{1 \mathrm{AB}}\right)$ & 9.7 & $9.5\left(\mathrm{P}_{\mathrm{L}, \mathrm{B}}\right)$ \\
\hline $\mathrm{PAE} \cdot \hat{a} \cdot \mathrm{P}_{\text {han,anax }}$ & $25 \%$ & $14 \%$ & $11 \%$ & N/A \\
\hline $\mathrm{P}_{\mathrm{S}, \mathrm{IT}}[\mathrm{ABm}]$ & 15.6 & 15.6 & 9.7 & 10.9 \\
\hline PAE $\alpha \mathrm{P}_{\mathrm{SAT}}$ & $25 \%$ & $25 \%$ & $11 \%$ & $8.8 \%$ \\
\hline IRR [dB] & $>20$ & $>20$ & $\mathrm{~N} / \mathrm{A}$ & $\mathrm{N} / \mathrm{A}$ \\
\hline $\mathrm{P}_{\mathrm{ne}} \cdot[\mathrm{m} \mathrm{W}]$ & $\begin{array}{l}220: \dot{a} 1 \mathrm{~V} \\
\text { (PA: 120) }\end{array}$ & $\begin{array}{l}23+(a-1 V) \\
\text { (PA: 134) }\end{array}$ & $\begin{array}{l}193(1)^{\circ} \\
\text { (PA: } 85 \times 2)\end{array}$ & $\begin{array}{l}186: 1 \mathrm{~V} \\
\text { (PA: } 114.6)\end{array}$ \\
\hline $\mathrm{P}_{\text {lat ans }} / \mathrm{P}_{\mathrm{DCC}}$ & $16.5 \%$ & $8.5 \%$ & $9.7 \% \%^{+}$ & $4.8 \%$ \\
\hline Area [mm²] & \multicolumn{2}{|c|}{$0.96 / 0.33$ (active) } & 0.28 (active) & 8 (only $T x$ ) \\
\hline Tech. & \multicolumn{2}{|c|}{$40 \mathrm{~nm}$ CNIOS } & $65 \mathrm{~nm}$ CMOS & $65 \mathrm{~nm}$ CNOS \\
\hline
\end{tabular}

Figure 9.5.6: Performance summary and comparison. 